http://dx.doi.org/10.18778/1505-9065.11.18

Vivien Bessières

Université de Limoges

vivien.bessieres@unilim.fr

\title{
HYPOTYPOSE LITTÉRAIRE ET INCRUSTATION BÉDÉIQUE : ESSAI DE STYLISTIQUE COMPARÉE
}

\begin{abstract}
"Hypotyposis in Literature and Insert in Comics: An Attempt of Comparative Stylistics"
SUMMARY - As a dynamic picture, the literary figure of hypotyposis seems consistent with the very definition of comics, series of drawings producing motion. However, if hypotyposis is always simultaneously descriptive and narrative, comics are on the contrary sets of descriptions which sequencing produces narration. In fact, a particular device of the ninth art matches more with the literary figure: the insert, that is the inlay of one or more panels within another. Automatically, the inserting panel becomes the background, the setting of a story developed in the foreground by the inserted panels. This device is not classical. It is to be found among the pioneers or the modern and contemporary authors, who use it, like hypotyposis, for epic purposes, or to give more life to a historical re-enactment, to confront collective with individual memory, or also to make several spaces flourish in the same time and several times flourish in the same place.
\end{abstract}

KEYWORDS - hypotyposis, comics, stylistics, esthetics, comparative literature, intermediality

"Hipotiposis literaria e incrustación en cómic: un ensayo de estilística comparada"

RESUMEN - En cuanto cuadro dinámico, la figura literaria de la hipotiposis parece concertarse con la propia definición del cómic, sucesión de imágenes generadora de movimiento. Sin embargo, si la hipotiposis es siempre simultáneamente descriptiva y narrativa, no ocurre lo mismo con el cómic, conjunto de descripciones que, por su sucesión, generan narración. De hecho, lo que mejor corresponde a la figura literaria es más bien un dispositivo particular del noveno arte: la incrustación, es decir la inscripción de una o varias viñetas dentro de otra. Automáticamente, la viñeta-marco se convierte en el trasfondo, el decorado de un relato desarrollado en primer plano por las viñetas incrustadas. Este procedimiento de incrustación no es clásico. Lo encontramos en los pioneros, o ente modernos y contemporáneos, que se sirven de él, a ejemplo de la hipotiposis, con fines épicos, o para dar mayor vida a una reconstrucción histórica, o bien para confrontar memoria colectiva y memoria individual, o bien incluso para que proliferen en un mismo tiempo varios espacios y en un mismo espacio tiempos diversos.

Palabras Clave - hipotiposis, cómic, estilística, estética, literatura comparada, intermedialidad

Description dynamique, tableau vivant, en action, l'hypotypose semble une figure littéraire difficilement transposable à la bande dessinée, tant c'est le neuvième art dans son ensemble qui vérifie a priori la définition : la bande dessinée, c'est cette peinture, ce tableau qui se dédouble autant de fois qu'il y a de vignettes, et qui entre pour ainsi dire en action, c'est ce récit qui se lit au présent du regard - toutes définitions qui conviennent bien aussi, en première analyse, à la figure de l'hypotypose. Dès lors, il devient bien hasardeux de vouloir parler d'hypotypose pour un dispositif spécifique de la bande dessinée. 
Pourtant, avant de baisser les bras, il conviendrait de se faire une idée plus précise de l'hypotypose littéraire elle-même. À prendre par exemple le récit de Théramène sur la mort d'Hippolyte, dans la Phèdre de Racine $(\mathrm{V}, 6)$, ou bien la résurrection de la chair prophétisée par les Tragiques d'Agrippa d'Aubigné (VII, 661-684), que trouve-t-on dans ces textes de si particulier, que l'on ne retrouve pas dans d'autres descriptions (ou narrations) ? Un premier test opératoire paraît être l'énallage temporelle et spatiale : le passage d'un temps délocuté comme le passé simple ou l'imparfait à un temps embrayé, le présent (de narration), ainsi que le passage d'un régime de référence anaphorique à de la deixis - traduction linguistique, stylistique de cet effet «devant les yeux », «ici et maintenant» relevé par la plupart des définitions de la figure. Ce n'est pas un hasard si les épopées et chansons de geste en sont friands : en des temps d'avant le cinéma, il faut de tels embrayages aux aèdes et bateleurs pour présenter la scène devant les yeux des auditeurs.

Mais qu'est-ce qui est vraiment embrayé, « présentifié » dans l'hypotypose ? Une description? Une narration? Les avis, les définitions semblent ici diverger. C'est peut-être que justement l'hypotypose est une description qui s'agite ou une narration qui prend la pose (et la pause) - une figure à la croisée de ces deux types de texte (narratif et descriptif) : le récit s'arrête sur un moment important, se met en scène, s'attarde sur certains détails révélateurs, les grossissant comme à la loupe ; la description s'anime, enchaîne les verbes d'action, dans un style asyndétique - le tout au service d'une émotion décuplée et de thèmes souvent violents comme la mort ou la guerre. L'hypotypose fonctionne ainsi comme un «opérateur d'intensification » pour reprendre l'expression de Jean-Michel Adam ${ }^{1}$.

Ce qui nous semble au cœur aussi de la figure, c'est ce curieux effet produit par un embrayage temporel et spatial, qui se double d'un effacement des embrayeurs de la première personne : la scène est au présent du regard, et en même temps le narrateur / descripteur s'en absente, c'est-à-dire qu'il y a énallage du passé (ou du futur) au présent, mais aussi de la première personne à la troisième. C'est très clair dans la prophétie des Tragiques, où le poète s'efface systématiquement de l'hypotypose pour lui donner le caractère d'une vérité prophétique que l'énonciateur se contenterait d'enregistrer d'un œil objectif : «C'est fait, Dieu vient régner...»

À partir de cette définition plus approfondie de l'hypotypose littéraire, il est possible de comprendre ce qui la distingue de la bande dessinée en général et ce qui la rapproche en même temps d'un dispositif particulier propre à cet art.

En effet, si la bande dessinée a bien quelque chose d'une narration descriptive ou d'une description narrative, elle n'est pas exactement tout cela à la fois : chaque vignette est en elle-même une sorte de description, de tableau, et c'est l'enchaînement, la séquence des vignettes qui produit du récit. Il est possible de

\footnotetext{
${ }^{1}$ J.-M. Adam, La Description, Paris, PUF, « Que-sais-je ? », 1993, p. 36.
} 
prendre chaque vignette à la fois pour ce qu'elle est en elle-même (une image arrêtée, picturale) et pour ce qu'elle est par rapport aux autres (un instant dans une chaîne narrative, une chaîne d'événements). Chaque vignette est les deux à la fois, mais certaines vignettes se situent davantage du côté de la narration, et d'autres du côté de la description.

Les premières se hissent par divers moyens hors de la séquence narrative : ce peut être, par exemple, un luxe de détails, l'exposition d'un nouveau lieu, l'absence ou la pose / pause des personnages, un cadre plus grand, un plan d'ensemble, pour créer un effet tableau, ou au contraire un cadre plus petit, un gros plan, pour étudier comme à la loupe un élément particulier ou mettre en avant une émotion. Le second type de vignette, narratif, s'insère dans la séquence par divers moyens opposés : une économie des détails, des décors à l'arrière-plan et des personnages au premier, omniprésents et agissants, un cadre de dimension classique, des plans moyens ou américains favorables à la représentation des actions.

Une fois cette distinction reconnue, bien sûr toute relative et susceptible d'hybridations diverses, il devient possible de faire apparaître un dispositif particulier où se télescopent, dans une seule vignette, ces deux principes - un peu comme l'hypotypose fait se télescoper description et narration dans le présent du regard.

Cela ne peut avoir lieu dans une vignette à dominante descriptive, qui fige un moment et un espace. Cela ne peut avoir lieu non plus dans une vignette à dominante narrative, qui laisse le décor à l'arrière-plan pour se focaliser sur les personnages. Ce qu'il faudrait, c'est une vignette qui soit exactement les deux en même temps, narration et description, à égalité - une vignette qui réussisse ce tour de force de ne pas cantonner la description à l'arrière-plan, en amont, tout en y laissant évoluer une action, des personnages.

Or, il semble bien qu'il existe un tel dispositif en bande dessinée, un dispositif que l'on appelle l'incrustation. Il consiste à inscrire dans une grande vignette une ou plusieurs autres plus petites. Une vignette descriptive classique est une grande vignette occupant plusieurs bandes, voire toute la planche, et représentant tel ou tel décor. À la rigueur, on trouvera au premier plan des personnages, souvent tout petits, mais l'ensemble n'en gardera pas moins une tonalité descriptive : c'est le décor qui importe avant tout dans cette case. Mais si une ou plusieurs vignettes sont incrustées à l'intérieur de cette première vignette descriptive (par exemple, les personnages en gros plan évoluant dans le décor, discutant, ou se poursuivant, ou tout ce que l'on voudra), alors, le dispositif ainsi créé n'est ni prioritairement descriptif, ni prioritairement narratif - mais les deux à la fois. En d'autres termes, l'incrustation empêche la vignette englobante d'être essentiellement descriptive : elle troue la description avec de la narration.

Bien sûr, l'hypotypose suppose une certaine longueur et densité pour opérer l'intensification qui la caractérise, tandis que l'incrustation peut se réduire à une 
seule vignette à l'intérieur d'une autre. Il n'en reste pas moins vrai que les deux procédés se rapprochent en bien des points.

Comme l'explique très bien Thierry Groensteen dans son Système de la bande dessinée $e^{2}$, il s'agit de couper court au principe de la succession classique des vignettes, fondé sur une articulation temporelle de type "puis », au profit d'une relation oppositionnelle de type « cependant». L'incrustation suppose formellement un contraste, une confrontation, voire une violence, car elle vient trouer l'image première, incrustante, pour y superposer une image seconde, incrustée. À partir de ce trait contrastif, une multitude de rapports spécifiques sont possibles : par exemple, champ et contrechamp, regard d'un personnage et scène qu'il contemple, narrateur et récit (ou souvenir, rêve, fantasme), observateur et scène observée, début et suite de l'action, déroulement et aboutissement de l'action, plan d'ensemble et gros plan, lieu global et détails révélateurs - et autres relations métonymiques de ce type. Pierre Fresnault-Deruelle évoque aussi « une sorte d'effet de loupe $»^{3}$ permis par l'incrustation, qui correspond bien à l'effet « devant les yeux » de l'hypotypose.

Mais tous ces cas sont-ils semblables ? Sommes-nous à chaque fois dans quelque chose qui ressemble à de l'hypotypose ? A priori, ce qui se rapproche surtout de cette figure littéraire, c'est l'incrustation de petites vignettes de récit dans une vignette englobante à dominante descriptive. On peut cependant avancer l'hypothèse que toute vignette qui en englobe d'autres se retrouve toujours à l'arrière-plan (en tant que décor) de quelque chose qui est mis au premier plan (en tant que personnage), sans que l'un ou l'autre des plans ne soit a priori privilégié.

En fait, les mots de «personnage » et de «décor» ne sont pas à prendre au pied de la lettre : dans une vignette englobante, tout peut faire office de décor, même un personnage ; dans une vignette incrustée, tout peut faire office de personnage, même un décor - exactement comme le gros plan au cinéma est, pour Gilles Deleuze ${ }^{4}$, toujours un gros plan de visage, c'est-à-dire qu'il traite ce qu'il cadre comme un visage, même si ce n'en est pas un. En d'autres termes, si, par exemple, une vignette englobante représente un personnage qui parle et la vignette incrustée le lieu qu'il décrit, le personnage se met à fonctionner comme un décor, un arrière-plan, et le lieu comme un personnage, un premier plan.

Cet exemple théorique soulève une autre difficulté. L'hypotypose est censée faire disparaître le narrateur, mettre la scène sous les yeux du lecteur / auditeur sans la médiation d'un énonciateur. Or, ici, elle fait justement apparaître le narrateur, comme dans toutes les incrustations où un personnage se met à raconter quelque chose. Et le problème est le même quand l'incrustation concerne un décor et celui qui le regarde.

\footnotetext{
2 T. Groensteen, Système de la bande dessinée, Paris, PUF, «Formes sémiotiques », 1999, p. $100-106$.

${ }^{3}$ P. Fresnault-Deruelle, La Bande dessinée, Paris, Armand Colin, 2009, p. 77.

${ }^{4}$ G. Deleuze, L'Image-mouvement. Cinéma 1, Paris, Les Éditions de Minuit, « Critique », 1983, p. 132.
} 
Dans une bande dessinée classique (sans incrustation), ce type de situations serait décomposé : dans une première vignette, le personnage se mettrait à raconter ou regarder ; dans la vignette suivante, on aurait ce qu'il raconte ou regarde. Le narrateur, le point de vue seraient clairement définis, délimités dans une vignette spécifique. Quel est l'effet provoqué par l'incrustation du narrateur ou du personnage-point de vue ? Les instances énonciatives ne sont plus distinctes de ce qu'elles énoncent. Ce faisant, un effet de télescopage se produit : le lecteur a à la fois, dans un même temps et un même espace, l'énonciateur et son énoncé. Autrement dit, le lecteur de bande dessinée accède en même temps que l'énonciateur au contenu de son énoncé : il n'y a pas de temps ni d'espace d'attente (tel le blanc inter-iconique traditionnel), l'identification est presque totale et le sentiment de présence, voire d'urgence, s'en trouve a priori, comme dans l'hypotypose, augmentée.

De même, ce style asyndétique typique de l'hypotypose, où l'énumération de détails, de mouvements révélateurs a un effet quasi-hallucinatoire sur le destinataire, pris dans la verve de la description en acte, se retrouve elle aussi, à certains égards, dans l'incrustation. Tout ce qui servait, dans la bande dessinée de facture classique, à assurer la liaison entre les vignettes (blancs inter-iconiques, récitatifs avec connecteurs de toutes sortes - «puis », «mais », «pendant ce temps », etc.), est ici réduit à sa plus simple expression parataxique. Mais bien sûr, c'est quand les vignettes incrustées sont nombreuses que ce style asyndétique est le plus évident.

Sauf erreur, l'incrustation n'est pas un dispositif classique, que ce soit dans la ligne claire franco-belge, dans les comics américains de l'âge d'or ou dans le manga japonais classique initié par Osamu Tezuka. On la trouve plutôt chez les pionniers comme Winsor McCay, ou à partir des années soixante, quand la bande dessinée entre dans son âge adulte, expérimentant de nouvelles formes et de nouveaux thèmes (le sexe et le sang).

C'est dire que l'incrustation fait un peu violence à la grammaire traditionnelle du neuvième art, de même qu'elle provoque elle-même une certaine violence en trouant une image au moyen d'une autre. Il n'est pas étonnant, dès lors, qu'elle soit souvent utilisée au service de thèmes comme la mort, le meurtre ou la guerre, à l'instar de l'hypotypose. C'est qu'il s'agit à chaque fois de raconter et de décrire une scène saisissante, émouvante, et donc forcément empreinte d'une forme de violence, réelle ou symbolique.

Winsor McCay, avec Little Nemo in Slumberland ${ }^{5}$, pose les bases d'un dispositif qui sera oublié pendant le classicisme avant d'être redécouvert (en même temps que Little Nemo) par les expérimentateurs modernes. Quasiment la moitié des aventures (et donc des planches) de Little Nemo se termine par une telle incrustation, où la représentation du petit héros dans son lit (ou tombé hors de son

\footnotetext{
${ }^{5}$ W. McCay, Little Nemo in Slumberland, New York Herald / New York American, 15 octobre 1905 - 26 décembre 1926 ; Little Nemo, 1905-1914, Taschen, 2000.
} 
lit) vient mettre un terme abrupt, trancher comme un couperet le rêve merveilleux qui s'était déployé, avec toute la verve que l'on connaît, dans les vignettes précédentes.

Que l'enfant soit content de se réveiller (parce que le rêve était un cauchemar) ou bien malheureux (parce qu'il a fait de beaux rêves), la dernière vignette vient s'incruster comme une violence à l'intérieur du rêve, à l'instar des parents du héros, qui apparaissent souvent dans cette dernière case, pour mettre fin au sommeil et réinstaurer le principe de réalité - s'ils n'apparaissent pas en chair et en os, ils le font par le biais de bulles de dialogues injonctives, s'incrustant au carré dans la vignette incrustée. Il s'agit bien alors toujours d'une chute, sur de multiples plans : chute hors du rêve, accompagnée souvent d'une chute hors du lit, et traduite par la chute d'une vignette à l'intérieur d'une autre, les pieds (du réel) dans le plat (du rêve) - le tout produisant une chute narrative, un dénouement de l'histoire, à la toute fin de la planche, en bas à droite.

Pour reprendre une idée de Tristan Garcia $^{6}$, une bande dessinée représente toujours un peu les contours rassurants d'une chambre d'enfant où le lecteur se réfugie pour échapper à la réalité adulte. Cet art est né au moment même où l'enfant acquérait une première forme d'indépendance sociale, et, concrètement, une chambre à lui seul où les parents n'étaient plus forcément les bienvenus. Little Nemo illustre idéalement cette nouvelle donne culturelle, et l'incrustation de la vignette tombée du réel dit bien la violence faite par ces parents (ce réel adulte) qui s'incrustent dans la chambre (lieu des rêveries enfantines). On trouve d'ailleurs souvent des signes avant-coureurs de cette incrustation terminale quand un nouveau personnage, un nouvel événement intervient dans le rêve de manière menaçante.

Mais, si l'on peut parler d'un événement violent, d'un réveil coupant court au rêve, en quoi peut-on encore parler ici de description ? En quoi le dispositif rappelle-t-il l'hypotypose ? De deux façons, semble-t-il. D'une part, parce qu'il traduit parfaitement cette sensation du rêveur d'être tout entier dans son rêve, cet effacement énonciatif dont il est la victime inconsciente : l'incrustation rend bien compte de ce télescopage entre deux plans de réalité et d'énonciation, d'autant que Winsor McCay joue très souvent avec la correspondance entre la posture du corps de Little Nemo dans le rêve et celle qu'il a dans son lit (jusque dans ces chutes, qui ont lieu sur les deux plans). D'autre part, la vignette englobante qui sert de support à l'incrustation est la plupart du temps l'une des plus grandes de la planche (quand la vignette incrustée est presque toujours la plus petite). Ces grandes dimensions permettent en général l'élaboration la plus grandiose, la plus picturale, descriptive du monde rêvé. Il s'agit donc toujours de faire entrer en

\footnotetext{
${ }^{6} \mathrm{~T}$. Garcia, «L'art des âges et des images », in : Les Têtes chercheuses [émission de radio], France Culture, 2016. Disponible sur : http://www.franceculture.fr/emissions/les-tetes-chercheuses/l-artdes-ages-et-des-images ; consulté le 21 juillet 2016.
} 
collision (ou collusion) de la description et de la narration, un monde à contempler et une chute dans le réel.

Dans la planche du 15 juillet $1906^{7}$, une princesse embrasse Little Nemo sur la bouche. Elle lui promet de lui montrer toutes les merveilles de son palais. Mais le méchant Flip demande au garde de l'aube d'envoyer le soleil. Dans la vignette englobante finale, le soleil éclaire de ses rayons un palais de Slumberland qui, paradoxalement, s'estompe, lui et ses habitants, dont la princesse qui venait d'embrasser Little Nemo. Ce dernier garde en revanche des contours très nets que l'on retrouve dans la vignette du réveil, à l'instar de sa position assise - il est le seul à ne pas disparaître. « Je n'ai pas envie de me lever », dit-il à la fin, et il semble en effet toujours dans son rêve. Si la vignette du rêve n'avait été que suivie de la vignette du réel, et non pas incrustée, l'effet de saisissement, de ravissement (ravissement amoureux et ravissement hors du rêve) aurait été perdu.

De l'autre côté du classicisme, dans la bande dessinée moderne et contemporaine, l'incrustation se met à refleurir un peu partout, dans des œuvres aux styles très divers. On en trouve beaucoup dans ce que Thierry Groensteen appelle le «nouveau baroque $»^{8}$ qui, à partir des années soixante-dix avec Philippe Druillet, se distingue notamment par « la multiplication des incrustations, l'accentuation maximale du contraste entre les grandes images faisant fond et les inserts ${ }^{9}$. Benoît Peeters se montre critique envers cette « utilisation décorative » de la planche :

Bien des effets d'allure spectaculaire, tels les incrustations de cases et les débordements hors des limites du cadre, obéissent à une logique de cet ordre : loin de correspondre à une véritable invention, ils fonctionnent souvent comme de simple dégradations de la continuité séquentielle, voire comme des compensations de sa pauvreté. Il n'est pas sûr, ainsi, que les bouleversements graphiques prodigués par bon nombre de comics des années 1980 et 1990 aient renouvelé en profondeur le rapport à la narration ${ }^{10}$.

Si l'incrustation n'a qu'une finalité décorative, il n'y a en effet plus grand chose à en dire. Mais cette utilisation peut aussi se doubler d'une « utilisation rhétorique », pour reprendre la typologie de Benoît Peeters. Dans les comics de Frank Miller, par exemple, les incrustations prolifèrent, au service d'une esthétique de la confrontation, de l'affrontement, voire du choc des civilisations. Ainsi, dans $300^{11}$, le dessinateur ne cesse de jouer avec ce pouvoir contrastif de l'incrustation, quand il raconte, sur une tonalité épique et apologétique, la bataille des Thermopyles où trois cent Spartiates ont résisté à deux cent mille Perses. Ses incrustations ont surtout deux fonctions. L'une, que l'on retrouve dans la composante

\footnotetext{
${ }^{7}$ W. McCay, op. cit., p. 48.

${ }^{8}$ T. Groensteen, Bande dessinée et narration. Système de la bande dessinée 2, PUF, «Formes sémiotiques », 2011, p. 48.

${ }^{9}$ Ibid.

${ }^{10}$ B. Peeters, Lire la bande dessinée, Paris, Flammarion, « Champs », 1998, p. 59.

${ }^{11}$ F. Miller, 300, Milwaukie (OR), Dark Horse Comics, 1998 ; trad. L. Darrow, Montreuil, Rackham, 1999.
} 
épique de l'hypotypose, est de pouvoir décrire à la fois une grande bataille (vignette englobante) et les mouvements, les corps à corps qui la constituent (vignettes incrustées). L'autre est de renforcer l'opposition entre des Perses très nombreux mais inertes et viciés (vignette englobante descriptive) et des Spartiates petits en nombre mais hommes d'action et de conviction (vignettes incrustées narratives).

Vers la fin, par exemple, Léonidas, le roi de Sparte, est acculé ${ }^{12}$. La vignette englobante, occupant toute la page, montre les Perses à gauche et sur les hauteurs (le roi Xerxès, les Immortels et le traître Ephialte), ayant tous dans leur ligne de mire Léonidas et ses 300 formés en tortue. En bas de la page, plusieurs petites vignettes sont incrustées. Elles se suivent et créent un récit qui développe la situation initiale montrée par la case incrustante : Léonidas commence par poser son casque, puis son bouclier, puis sa lance et finit par s'agenouiller. On verra dans la planche suivante qu'il ne s'agissait pas de se rendre mais seulement de se libérer d'un armement trop encombrant pour pouvoir lancer avec le plus de précision possible un trait sur Xerxès. Léonidas fait de nécessité vertu : enfermé dans les faibles dimensions des vignettes incrustées, il se fait encore plus petit, plus léger, pour défaire la boursouflure perse.

À rebours d'une telle utilisation, qui verse souvent dans le décoratif ou l'idéologique, on peut évoquer l'usage modéré et dialogique qu'en fait François Bourgeon dans ses séries. Supposant toujours la mise en arrière-plan d'un décor d'ensemble traversé, au premier plan, par de l'action, l'incrustation se prête très bien, comme l'hypotypose, aux reconstitutions épiques et/ou historiques. On l'a vu avec 300 et c'est le cas aussi avec la bande dessinée historique telle que la pratique François Bourgeon, à ceci près que Les Passagers du vent ou Les Compagnons du crépuscule, pour prendre les deux grandes séries de l'auteur, mettent ce dispositif au service d'une confrontation qui se refuse à faire pencher la balance d'un seul côté en faisant fi de toute réalité historique. L'incrustation ne confronte pas un groupe (vignettes incrustées) à un autre (vignette englobante) comme dans 300 , mais plusieurs personnages de tous bords et horizons (vignettes incrustées) à l'intérieur d'une vaste fresque attentive aux détails et à l'exactitude historique (vignette englobante).

Le premier tome des Passagers du vent (La Fille sous la dunette) ${ }^{13}$ s'ouvre de manière frappante sur une incrustation représentant, dans la vignette englobante de la taille d'une demi-planche, la voilure d'un navire de la Marine Royale, et, dans les deux vignettes incrustées, deux profils en gros plan d'un marin, à gauche, qui tend un couteau menaçant, et d'un albatros, à droite, qui semble le défier du regard. On retrouve en très petits, à l'intérieur de la vignette englobante, ces mêmes deux personnages perchés tout en haut d'une grande voile. Le récitatif

\footnotetext{
${ }^{12}$ Quand aucune référence de page n'est indiquée, c'est que l'album n'est pas numéroté, comme cela arrive souvent en bande dessinée.

${ }^{13}$ F. Bourgeon, Les Passagers du vent. Tome 1 : La Fille sous la dunette, Grenoble, Glénat, 1979.
} 
dit : «Entre le marin et l'albatros, il y a comme une très ancienne querelle ». Dans la vignette englobante qui suit, représentant la mer et le navire au loin, deux nouvelles vignettes incrustées nous montrent l'albatros qui s'envole et le marin qui tombe à l'eau. L'opposition n'est plus dissymétrique comme chez Miller, mais symétrique, dialogique (chacun a la même chance, la même portion d'espace), et elle s'effectue sur fond d'un vaste décor enchevêtrant culture (la voilure de la première vignette englobante) et nature (le vaste océan de la seconde).

Dès lors, à partir du genre privilégié de la reconstitution historique, l'incrustation est passée de la mémoire collective à la mémoire individuelle, en même temps que le style s'est singularisée selon les auteurs. On en trouve de nombreux avatars. Dans Maus d'Art Spiegelman, il y a cette case glaçante de quatre Juifs allemands pendus en 1942 "pour l'exemple» (parce qu'ils "vendaient sans ticket $\gg)^{14}$ : sur cette vignette englobante vient s'en incruster une plus petite où le personnage principal apprend la nouvelle en compagnie de deux proches - un récitatif annonce alors la vignette englobante : «Le lendemain, j'ai été rue Modrzejowska et je les ai vus... » Or, cette vignette incrustée troue l'englobante de manière à oblitérer le visage de deux des quatre pendus : le lecteur a l'impression fugitive que le personnage principal se substitue aux pendus, impression renforcée par le fait que tous les Juifs de ce témoignage sont dessinés en souris (et les nazis en chats). Si l'auteur avait utilisé une simple succession de vignettes, du temps serait réellement passé entre les deux, l'expression «le lendemain » aurait été respectée, mais le lecteur n'aurait pas eu cette impression d'urgence, de choc, comme si ces premiers pendus annonçaient déjà tous les morts à venir et la mort éventuelle, symbolique, du personnage principal - père de l'auteur, témoin de la Shoah. Il y a vraiment là comme une correspondance à l'énallage temporel typique de l'hypotypose : ce qui a normalement lieu « le lendemain » devient « ici et maintenant $»$.

En 1989, Richard McGuire dessine une bande dessinée expérimentale de six pages intitulée $H_{e r e}{ }^{15}$, qui fait de l'incrustation le principe moteur de son esthétique et qui aura une grande influence sur la création contemporaine, notamment chez Chris Ware ${ }^{16}$. La première vignette représente le coin d'une pièce vide, la seconde le même coin mais meublé et occupé par un couple qui attend un bébé - et elle est accompagnée d'un récitatif indiquant «1957». Sur toutes les vignettes suivantes, ce sera toujours le même endroit représenté. Dans la troisième, le père apprend à quelqu'un au téléphone que son fils vient de naître (toujours en « 1957 »). Dans la quatrième, encore la même année, la mère tient

\footnotetext{
${ }^{14}$ A. Spiegelman, Maus. Vol. 1, New York, Pantheon Books, 1986; trad. Judith Ertel, Paris, Flammarion, 1992, p. 83.

${ }^{15}$ R. McGuire, Here, Raw, vol. 2, 1989 ; Neuvième Art, $\mathrm{n}^{\mathrm{0}}$ 12, janvier 2006, p. 50-55. Ces six planches deviendront ensuite la matrice d'un grand roman graphique : R. McGuire, Here, New York, Pantheon Books, 2014 ; Ici, trad. I. Troin, Paris, Gallimard, 2015.

${ }^{16}$ Voir C. Ware, «Une appréciation reconnaissante », Neuvième Art, n ${ }^{\circ} 12$, janvier 2006, p. 48-49.
} 
son nouveau-né dans les bras et le père lui demande si elle a besoin de quoi que ce soit. Dans la cinquième, tout d'un coup, on passe à « 1922 » et une femme au téléphone répond «What ? » tandis qu'une autre attend à côté d'elle. Au milieu de cette vignette, la trouant en son centre, une autre vignette est incrustée et représente la mère avec son nouveau-né, en « 1957 », répondant à son mari qu'elle veut bien le biberon de l'enfant. À partir de là, les incrustations se multiplient de vignette en vignette et les âges se télescopent, depuis la préhistoire jusqu'au futur - mais toujours à partir du même endroit. Dès lors, le lieu se charge d'échos passés et à venir innombrables. Ici, le lecteur est confronté à une sorte d'hypotypose maximale, où ce n'est plus une même scène qui est vécue de manière intense, mais le lieu lui-même qui s'intensifie de toutes les scènes qu'il a vécues. Dès lors, si hypotypose il y a, c'est celle du temps lui-même plus que d'un événement dans le temps.

Inspiré par ce premier tour de force, Chris Ware utilise de même l'incrustation comme la matrice d'une nouvelle recherche du temps perdu. Développant un dispositif inauguré dans Jimmy Corrigan ${ }^{17}$, et finalement un peu à la manière de La Vie, mode d'emploi de Georges Perec ${ }^{18}$, Chris Warre utilise l'immeuble, dans Building Stories ${ }^{19}$, comme la vignette englobante de nombreuses grandes planches, à partir de laquelle s'incrustent, en un système complexe de flèches et de renvois, des vignettes partant d'un détail de l'immeuble pour développer un récit ancré dans la mémoire d'un des occupants. Il ne s'agit plus seulement de rendre vivante une scène unique, mais de faire foisonner un lieu de tout le récit, de toute la mémoire qu'il contient.

La perspective du dessin est axonométrique ${ }^{20}$, et non plus conique, c'est-àdire que l'image ne respecte pas la perspective oculaire traditionnelle, mais met à plat les décors comme dans un mode d'emploi, et selon un principe très proche de la délocution propre à l'hypotypose. Cette « délocution du regard» donne un caractère froid, objectif, technique au dessin, le rapprochant du diagramme. De plus, les vignettes incrustées sont disposées de telle sorte qu'elles peuvent se lire dans tous les sens et en commençant par n'importe laquelle - de la même manière que l'œuvre tout entière est constituée de quatorze livrets et opuscules de dimensions différentes, qui peuvent se lire dans tous les ordres possibles. Et Chris Ware y fait souvent parler l'immeuble lui-même, au moyen de nombreux récitatifs faisant office de narration première, comme si le point de vue principal n'était plus celui d'un regard, mais celui d'un lieu qui aurait comme Argos des yeux partout.

\footnotetext{
${ }^{17}$ C. Ware, Jimmy Corrigan, New York, Pantheon Books, 2000 ; trad. A. Capuron, Paris, Delcourt, 2002.

${ }^{18}$ G. Perec, La Vie mode d'emploi, Paris, Hachette, 1978.

${ }^{19}$ C. Ware, Building Stories, New York, Pantheon Books, 2012 ; trad. A. Capuron, Paris, Delcourt, 2014.

${ }^{20}$ Voir J. Samson, «Une célébration de la bande dessinée », in : J. Samson et B. Peeters, Chris Ware, la bande dessinée réinventée, Bruxelles, Les Impressions Nouvelles, « Réflexions faites », 2010, p. 101-158, ici p. 141.
} 
Cette disparition d'un point de vue particulier et d'un ordre régulateur, si froide en apparence, contribue paradoxalement à générer, à l'instar de l'hypotypose, une émotion esthétique intense, liée à l'écoulement du temps et à nos tentatives pour le juguler : l'incrustation permet ici de ne plus traiter le passé (ou le futur) comme du passé (ou du futur), mais comme un nouveau présent à égalité avec celui du récit premier, sans hiérarchie.

À l'instar de l'hypotypose, cette esthétique de l'objectivité est en même temps le véhicule d'une grande expressivité, d'un ravissement affectif du lecteur :

On peut aussi dire du graphisme de Ware qu'il tend à l'effacement de l'ego de l'artiste pour exprimer l'essence de la chose représentée de telle façon qu'il laisse la plus grande place à l'expressivité du média en tant que tel; le contraire donc de l'affirmation d'un style où la personnalisation graphique compte autant, sinon plus, que l'expression qu'elle sert ${ }^{21}$.

Le titre de l'œuvre donnait déjà les clés de cet immeuble graphique créé par Chris Ware. Building Stories, ce sont à la fois les « histoires issues d'un immeuble particulier » et le « fait de fabriquer, de manière architecturale (to build), des histoires » - tant il s'agit bien, avec la bande dessinée, de construire des histoires comme des maisons, de sorte qu'une image ne chasse pas obligatoirement la précédente, comme au cinéma, mais " s'étage " par rapport aux autres sans disparaître complètement de la vue du lecteur. Ce n'est pas pour rien que les stories du titre sont aussi le mot anglais pour désigner les « étages » d'un immeuble, étages qui renvoient irrésistiblement aux bandes de la bande dessinée. C'est ainsi que l'immeuble que l'on retrouve si souvent en incrustation dans les planches de cette œuvre fonctionne comme une métonymie de toute l'œuvre - une incrustation de l'œuvre à l'intérieur d'elle-même.

À la fin de ce rapide tour d'horizon des déclinaisons possibles de l'incrustation comme équivalent de l'hypotypose, il importe pour conclure d'ajouter qu'il ne s'agit pas pour nous de considérer à tout prix l'ensemble des incrustations de la bande dessinée comme des hypotyposes cachées, mais plutôt de contribuer à la légitimation du neuvième art en montrant qu'il peut être aussi sophistiqué que les arts de l'écrit, ainsi que de participer à la réflexion sur la représentation du temps dans les arts : en quoi chacun d'eux envisage toujours a priori la temporalité de manière très différente, en quoi chacun d'eux se lance toujours, in fine, à la recherche d'un même temps perdu.

\section{Bibliographie}

Adam, Jean-Michel, La Description, Paris, PUF, « Que-sais-je ? », 1993

Bourgeon, François, Les Passagers du vent. Tome 1 : La Fille sous la dunette, Grenoble, Glénat, 1979

Deleuze, Gilles, L'Image-mouvement. Cinéma 1, Paris, Les Éditions de Minuit, « Critique », 1983

${ }^{21}$ Ibid., p. 138. 
Fresnault-Deruelle, Pierre, La Bande dessinée, Paris, Armand Colin, 2009

Garcia, Tristan, "L'art des âges et des images », in: Les Têtes chercheuses [émission de radio], France Culture, 2016. Disponible sur: http://www.franceculture.fr/emissions/les-teteschercheuses/1-art-des-ages-et-des-images ; consulté le 21 juillet 2016

Groensteen, Thierry, Système de la bande dessinée, Paris, PUF, «Formes sémiotiques », 1999

Groensteen, Thierry, Bande dessinée et narration. Système de la bande dessinée 2, Paris, PUF, «Formes sémiotiques », 2011

McCay, Winsor, Little Nemo in Slumberland, New York Herald / New York American, 15 octobre 1905 - 26 décembre 1926 ; Little Nemo, 1905-1914, Taschen, 2000

McGuire, Richard, Here, Raw, vol. 2, 1989 ; Neuvième Art, $\mathrm{n}^{\circ}$ 12, janvier 2006, p. 50-55

McGuire, Richard, Here, New York, Pantheon Books, 2014 ; Ici, trad. Isabelle Troin, Paris, Gallimard, 2015

Miller, Fank, 300, Milwaukie (OR), Dark Horse Comics, 1998 ; trad. Lorraine Darrow, Montreuil, Rackham, 1999

Peeters, Benoît, Lire la bande dessinée, Paris, Flammarion, « Champs », 1998

Perec, Georges, La Vie mode d'emploi, Paris, Hachette, 1978

Samson, Jacques, «Une célébration de la bande dessinée », in : Samson Jacques et Peeters, Benoît, Chris Ware, la bande dessinée réinventée, Bruxelles, Les Impressions Nouvelles, «Réflexions faites », 2010, p. 101-158

Spiegelman, Art, Maus. Vol. 1, New York, Pantheon Books, 1986 ; trad. Judith Ertel, Paris, Flammarion, 1992

Ware, Chris, Jimmy Corrigan, New York, Pantheon Books, 2000 ; trad. Anne Capuron, Paris, Delcourt, 2002

Ware, Chris, «Une appréciation reconnaissante », Neuvième Art, $\mathrm{n}^{\circ}$ 12, janvier 2006, p. $48-49$

Ware, Chris, Building Stories, New York, Pantheon Books, 2012 ; trad. Anne Capuron, Paris, Delcourt, 2014

\section{Vivien Bessières}

Vivien Bessières travaille depuis peu à l'Université de Limoges comme maître de conférences en langue française (à partir du $1^{\text {er }}$ septembre 2016). Auparavant, allocataire-moniteur, puis ATER, puis vacataire à l'Université de Toulouse Jean Jaurès en linguistique, stylistique et littérature comparée (2007-2016), ainsi que professeur agrégé de lettres classiques au lycée (2013-2016). Thèse de lettres modernes dirigée par Jacques Dürrenmatt et soutenue à Toulouse 2 en 2011 avec les félicitations du jury: Antiquité et postmodernité. Les intertextes gréco-latins dans les arts à récit depuis les années soixante (fiction, théâtre, cinéma, série télévisée, bande dessinée). Version remaniée à paraître courant 2017 aux éditions Classiques Garnier. Publications diverses sur la présence de l'Antiquité dans les arts narratifs et sur les correspondances stylistiques possibles entre les langues et entre les arts. Se consacre ces dernières années à des questions de stylistique et de poétique du récit dans un contexte d'intermédialité et à la réflexion sur une stylistique comparée, par-delà la seule langue française et la seule littérature. 